\title{
Adoption of E-commerce Payment Systems by Commercial Banks in Kenya
}

\author{
Ndubi Jesse Nakhumwa \\ Head of Management Information Systems Department \\ Kul Graphics Ltd., Maasai Rd, Nairobi \\ P.O. Box 7 - 50409, Nambale - Kenya \\ E-mail: jnakhumwa@gmail.com
}

\begin{abstract}
E-commerce, which is combination of traditional commerce and Internet, has brought dramatic changes of the way business transactions are conducted prompting banks, as the intermediary financial instruments, to adopt and adapt electronic payment systems (EPS). These e-payment systems which include debit and credit cards, electronic fund transfer, mobile payments platforms and internet banking are already in use in Kenya market. Importantly to note is the fact that electornic payment instruments are not used with equal intensity even in developed countries due to various reasons. The research thus is focused on identifying key drivers for adoption of EPS in Kenya market by banks.
\end{abstract}

The researcher identified major variables affecting adoption of EPS which included security status, perceived level of trust, infrastructure capability to handle the system, marginal cost reduction and perceived associated benefits. A descriptive census survey of all the 43 banks was then done through a structured questionnaire. With aid of technology acceptance model and DeLone \& McLean Information System Success model, the data collected was empirically analysed and results presented.

With different intensity, the findings of the study revealed that many banks in Kenya are implementing EPS platforms. The driving forces for the adoption are the factors identified in the conceptual framework of this study. Bank respondents successfully did the rating of these factors. Therefore, the study recommends for a concerted effort amongst EPS key players to streamline operations in their area of concern. They should establish policies and legal framework good for electronic transactions as well as building sound telecommunication infrastructure countrywide. Again, this study is just but a stepping-stone to a better analysis that will unlock the potential of e-payment systems. The researcher encourages both academicians and practitioners to critique the study findings.

\section{Indexing Terms/Keywords}

E-commerce, Electronic payment systems, Information Technology in banks, Electronic Money, Systems Acceptance

\section{Academic Disciplines}

Information Science, Management Information Systems, Information Systems, Computer Science, Financial Management Information Systems.

\section{Subject Classification}

Library of Congress Classification

Type (Method/ Approach)

Literary Analysis, Survey

\section{Council for Innovative Research}

Peer Review Research Publishing System

Journal: International Journal of Management \& Information Technology

Vol. 9, No. 2

editor@cirworld.com

www.cirworld.com, member.cirworld.com 


\section{INTRODUCTION}

The birth of information and communication technology (ICT) as a result of merging of computer science and telecommunication engineering, brought dramatic changes of the way business is conducted to compete in the market place and spread throughout the globe (Schneider, 2011). The combination of traditional commerce and Internet, providing opportunities for business or organizations to develop new business models to take advantages of globalization is known as electronic commerce or e-commerce.

Chaffey, (2009) describes e-commerce as all electronically mediated information exchanges between an organisation and its external stakeholders. That means e-commerce includes other activities, "such as businesses trading with other businesses and internal processes that companies use to support their buying, selling, hiring, planning, and other activities" (Schneider, 2011; Zwass, 2003). Therefore e-commerce involves digitally enabled commercial transactions between and among organizations and individuals with exchange of value across boundaries while ebusiness is the digital enablement of transactions and processes within a firm (Chaffey \& Wood, 2005).

Kalakota and Whinston (1997) cited by Chaffey \& Wood (2005) structures e-commerce into three categories, i.e., business-to-consumer (B2C) such as Amazon.com and travelocity.com offering online shop where products/ services are sold from company to end user; business-to-business (B2B) such as PerfectCommerce.com and Grainger.com where products and services are sold from one company/ organisations to another, and consumer-to-consumer (C2C) such as Half.com and eBay.com where products and services are sold by consumers to fellow consumers through a third party who charges a flat rate. However, not all products and services can be sold on the internet a part from those that take advantage of the convenience of internet such as computer software, online books, travel and hotel bookings, among others.

ICT has made it possible to have electronic payment systems like debit cards, credit cards, electronic fund transfer, direct credits and internet banking. E-payment can refer to a payment system for buying and selling goods or services offered through the internet or any type of electronic fund transfer. Banks play a critical role in these e-payments as an intermediary. Traditional e-payment systems such as MoneyGram and Western Union are noted to have many limitations which inhibit consumers from adopting them. Earlier research suggests that some of these factors relate to lack of trust, security, usability, high transaction costs, lack of perceived advantage and perceived risk. These factors are deemed to be important to provide banks with the confidence to switch to an online payment system (Ozkan, 2010).

Importantly to note is the fact that electornic payment instruments are not used with equal intensity even in developed countries. The variations in intensity of adoption as revealed by previous research works are caused by issues to do with security, infrastructure, regulatory and legal and socio-cultural challenges (Ingenico, 2012). However, efficient and safe payment systems matter for the smooth functioning of commerce, financial intermediation and ultimately economic growth.

The popularity of e-payment systems is enhanced with widespread use of internet based shopping and banking (Bizina, 2012). Nevertheless, electronic payments systems innovations are meant to fulfill two perspectives: - One is to replace existing funds transfer systems that are deemed risky because of their "informality" therefore freeing people from money lenders or other shady characters (a version of the empowerment). The second context, is that the designers of electronic payment systems are simply seeking a piece of the money transfer business (a version of the market share), where they seek to replace Western Union or MoneyGram (Boyd \& Jacob, 2007).

E-commerce provides the opportunity to buy and sell products, information and services on the internet. Thus it requires an effective standardised online payment system. According to Đurić, Marić, \& Gašević (2007), several online payment proposals both for coin-like and cheque-like systems have arisen with none achieving mass acceptance. This lack of a uniform platform for operation by banks leaves a strategic linger. All the e-payment service providers are expected to meet five central requirements of electronic payments to win trust from commercial sectors, i.e., security, cost, time, risk and capacity. Among the five factors, security is very crucial as it affects the trust and confidence of customers.

The other risks with e-payment lies in banks over reliance on IT; increased electronic access by customers and attacks by hackers through packet/ address spoofing, stealth diagnosis, sniffers, sweepers and backdoors; low public acceptability, lack of adequate infrastructure, staff resistance and legal challenges.

Laudon \& Traver $(2007)$ highlights the advantages of electronic payment systems over the traditional methods. They encourage privacy, integrity, compatibility, good transaction efficiency, acceptability, convenience, mobility, low financial risk and anonymity. The other advantages are reaching out to customers in remote zones, minimizing on costs associated with premises leasing and security and enhancing customer awareness and loyalty (Magutu, et al., 2011).

Kenya's electronic payment systems dates back to 2005 when Central Bank of Kenya commissioned a Kenya electronic payment and settlement system (KEPSS), a pioneer real time gross settlement (RTGS) system. RTGS supports continous concurrent processing and final settlement of funds transfer instructions from one bank to another in the accounts of participants in the Central Bank of Kenya as long as they have sufficient covering balance or credit. The other electronic payment systems in use include Nairobi automated clearing house (NACH), ATMs using cards, securities payment and settlement systems (Central Depository \& Settlement Corporation), cross border money 
transfers including Western Union and MoneyGram, and mobile payments such as M-pesa, Yu Cash, Orange Money and Airtel Money (CBK, 2012).

Since electronic payments are typically cheaper than paper-based or cash payments, pricing these transactions should speed up the shift to electronics (Bolt, Humphrey, \& Uittenbogaarda, 2008). The key players in Kenya electronic payment systems in conjunction with Central Bank of Kenya are banks, infrastructure providers, non-bank mobile service providers, and regulatory bodies including the government.

\section{FRAMEWORK FOR THE LITERATURE REVIEW}

Two research theories were adopted for this study, that is, technology acceptance model (TAM) and DeLone \& McLean Information System Success model. Most of the studies touching on electronic payments have their roots in Technology Acceptance Model (TAM) originally proposed by Davies in 1986. TAM was designed to predict user's acceptance of Information Technology and usage in organisational context as shown below.

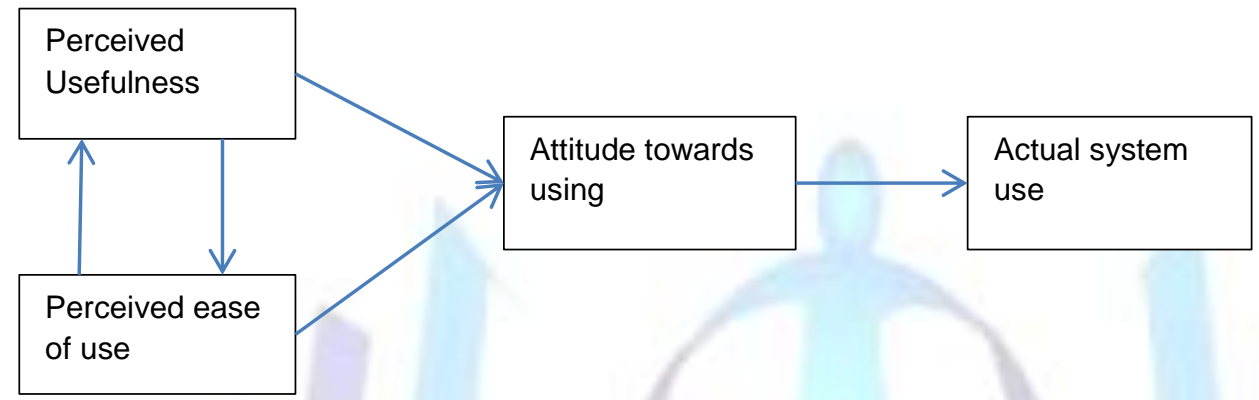

Figure 1: Technology Acceptance Model (TAM)

Source: Lule, Omwansa, \& Waema (2012)

TAM model suggests that two important factors influence users when confronted with a new technology. These factors presented below determines when and how users will use the new technology (Lule, Omwansa, \& Waema, 2012). They are: -

A. Perceived usefulness - The degree to which an individual believes that using the system will help him or her to attain gains in job performance, and

B. Perceived ease of use -The degree of ease associated with the use of the system

Technology acceptance model was therefore used to explore the level of motivation and user attitude that determined whether the user actually used or rejected the system in question (Kim, Mannino, \& Nieschwietz, 2009).

On the other hand, the use of DeLone \& McLean Information System Success model as a framework for measuring the success or effectiveness of information systems, was critical to understanding of the value and efficacy of information systems management actions and relevance of its investments (Delone \& McLean, 2003). Key factors in considerations are identified in the figure below.
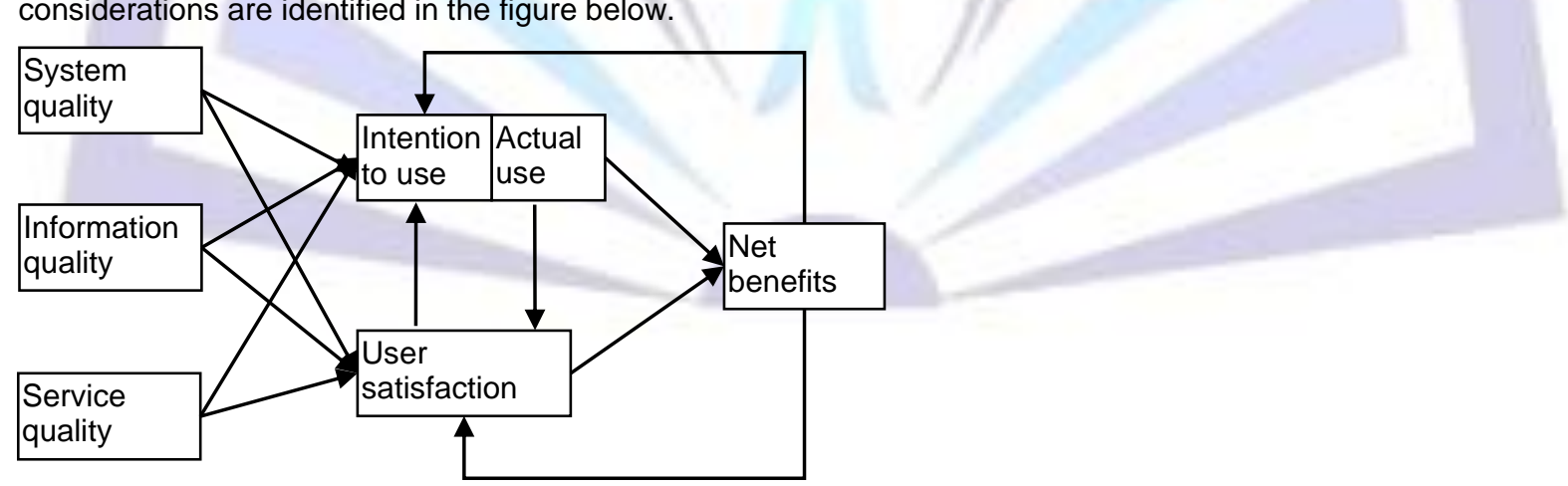

Figure 2: Delone \& McLean IS Success Model

Source: Delone \& McLean (2003)

A. System quality - this refer to the features that you expect from a system mainly reliability, portability, user friendliness, understandability, effectiveness, maintainability, economy, and verifiability.

$B$. Information quality - refers to the quality of information outputs, that is, management reports and web pages. It is a key dimension of end-user satisfaction instruments.

C. Service quality - a part from the use of SERVQUAL rater, other measures of service quality include the skill, experience, and capabilities and responsiveness of the support staff.

D. System use - is the degree and manner in which staff and customers utilize the capabilities of an information system. Empirical studies have adopted multiple measures of information system use, including intention to use frequency of use, self-reported use, and actual use. 
E. User satisfaction - The most widely used user satisfaction instruments are the Doll et al. (1994) End-User Computing Support (EUCS) instrument and the User Information Satisfaction (UIS) instrument (Petter, DeLone, \& McLean, 2008).

F. Net benefits - Different aspects of impact, i.e., task productivity, task innovation, customer satisfaction, and management control are the key dimensions measured at the individual level. At the organizational level, a variety of measures are employed; but profitability measurements seem to be preferred (Petter, DeLone, \& McLean, 2008).

A combination of two theories generated a framework that provided a better study platform for understanding the drivers explaining why users adopt e-commerce payment systems and whether the adopted systems had any business relevance. This operationalised construct is shown below.

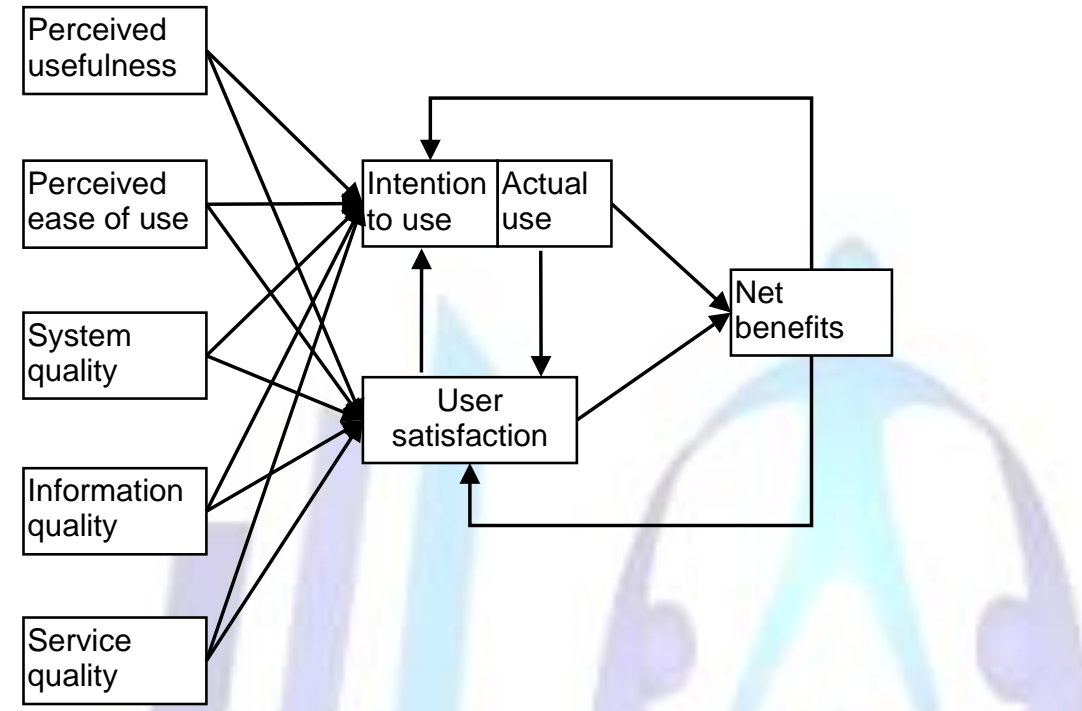

Figure 3: Research Constructs for Adoption and Impacts of Information Systems Source: Researcher (2013) Adopted from Delone \& McLean (2003)

\section{RESEARCH METHODOLOGY}

A descriptive cross-sectional survey research design was adopted to offer a substantial body of knowledge about extent of online payment systems and drivers for adoption of this methods of payament by banks in Kenya. The descriptive approach was concerned with who, what, where, when or how much of the interest variables being studied were adopted by banks (Howitt \& Cramer, 2011). This approach was prime for investigating the forces behind the success or failure of electronic payment systems. A survey strategy was adopted since it allows collection of a large amount of data from a sizeable population in a highly economical way. Quantitative data collected with survey are easy to analyse quantitatively using descriptive and inferential statistics. The results thus can be used to suggest possible reasons for particular relationships between variables and to produce models of these relationships. For the case of this research, an establishment of relationship between the extent of EPS used by banks against the drivers for adoption of EPS was very important.

As at 31st December 2012, the banking sector comprised of the Central Bank of Kenya, as the regulatory authority, with other 43 commercial banking institutions (CBK, 2012). Out of the 43 banking institutions, 30 are locally owned banks while 13 are foreign owned. Since there are only 43 banking institutions, the study covered the whole sample frame (population). With census, generalisation of results is made simpler and accurate (Saunders, Lewis, \& Thornhill, 2009).

Although the study coverered all the banks in Kenya as census, the subjects of interest were two staff members from each bank, that is, one from finance and another from information and communication technology. The people chosen for the study bore characteristics of interest to the theoretical concerns of the researcher, hence forming a research sample set. Based on the facts stated in the preceding paragraphs, the respondents in this study were arrived at as follows:

$\mathrm{N}($ Total number of banks in Kenya $(\mathrm{CBK}, 2012))=43$

$\mathrm{n}=\mathrm{N} \times 2->43 \times 2$ respondents from each bank $=86$

Therefore $\mathrm{n}=86$

Out of the 86 questionnaires given out to respondents, 70 were successfully returned with 16 failing to be honoured. This represents $81 \%$ response rate. According to Saunders, Lewis, and Thornhill (2009), a questionnaire response rate of between $50-70 \%$ is adequate for research study. 
Primary data was collected using structured questionnaires that focused on on benefits of deploying e-payment systems, the challenges that come with e-payment system and lastly the key drivers that were behind adoption of epayment systems irrespective of the challenges that were faced by banks. The questionnaire was validated through a rigourous process.

The following Linear Probability Model (LPM) was used to establish the drivers of e-commerce payment systems.

$$
Y=\beta_{0}+\beta_{1} X_{1}+\beta_{2} X_{2}+\varepsilon
$$

Whereby $\quad \mathrm{Y} \quad=$ e-payment methods (ATM, debit/credit cards, mobile, and Internet)

$\beta_{0 .} \beta_{2}=$ Constant/ co-efficient

$\mathrm{X}_{1} \quad$ = Determinants of e-payment (security, trust/risk, capacity, cost, benefits)

$\mathrm{X}_{2}=$ Characteristics of the bank (number of branches and ownership)

$\varepsilon \quad=$ Random error term

\section{DATA ANALYSIS, RESULTS AND DISCUSSION}

Analysis of data looks for patterns or trends across the results, to track progressions or to seek out repetition of certain results to build up a strong case. More so, quantitative analysis deals with data in the form of numbers and uses mathematical operations to investigate their properties (Walliman, 2011).

To be sure that what was found in the questionnaires actually represented what was measured, a section of the questionnaire was designed to capture background data on respondents as well the banks they represented. Background data therefore took care of the validity and reliability of the questionnaires used as data collection tools.

\subsection{Extend of EPS Use by Kenya Banks}

Banks and other financial institutions in Kenya are one of the largest investors in the fields of information systems (IS), and there are many indications that these trend will continue in the future. When the correspondents were asked whether their respective banks use electronic payment systems, 91\% percent admitted having installed ATMs, 64\% gave consent of using debit cards, credit cards attracted $73 \%$, mobile payment methods got $88 \%$ with internet supported transactions taking $73 \%$. All the five methods of electronic payments under study scored above $50 \%$ which is an indicator of their intensity of use by commercial banks in Kenya. Table 4.4 below indicates the extent of adoption of electronic payment systems by commercial banks in Kenya.

Table 1: Extent of EPS Adoption by Banks in Kenya

\begin{tabular}{|l|r|r|r|r|r|r|}
\hline & \multicolumn{2}{|c|}{ No } & \multicolumn{2}{c|}{ Yes } & \multicolumn{2}{c|}{ Total } \\
\cline { 2 - 7 } & Count & Row N \% & Count & Row N \% & Count & Table N \% \\
\hline Bank e-system ATM & 6 & $9 \%$ & 64 & $91 \%$ & 70 & $100 \%$ \\
\hline Bank e-system debit Cards & 24 & $36 \%$ & 42 & $64 \%$ & 66 & $100 \%$ \\
\hline Bank e-system Credit Cards & 17 & $27 \%$ & 47 & $73 \%$ & 64 & $100 \%$ \\
\hline $\begin{array}{l}\text { Bank e-system Mobile } \\
\text { Payment }\end{array}$ & 8 & $13 \%$ & 56 & $88 \%$ & 64 & $100 \%$ \\
\hline $\begin{array}{l}\text { Bank e-system Internet } \\
\text { Payment }\end{array}$ & 17 & $27 \%$ & 46 & $73 \%$ & 63 & $100 \%$ \\
\hline
\end{tabular}

The most preferred method of electronic payment by banks is ATM (91\%) followed by mobile payment methods $(80 \%)$. Debit card payment method was the least accepted by correspondents with a rating of $(60 \%$ each). The bar chart allows direct comparison of the methods of payment against the opinions of the respondents.

Kenya as a developing country still has a long way to go before the banks can accept implementing electronic payment systems as a mainstream method of financial transaction. The variations in intensity of adoption as revealed by previous researches are security, infrastructure, regulatory and legal issues and socio-cultural challenges (Ingenico, 2012). 


\subsection{Benefits of Electronic Payment Systems}

The benefits of electronic payment systems were sought from the respondents and their response is shown in the table below. ATM methods of payment had a strong approval of $15 \%$ probability for increasing global reach of bank payments while $29 \%$ agreed that the ATM service would grant their clients a 24/7 hours service without the attention of teller clerk. Internet payment method also attracted a probability of $31 \%$ for improving the customer access through low capital costs as well as providing a $24 / 7$ hours service to the customers. However, $20 \%$ of respondents did not agree that internet payment adds value to global reach. This could be explained by the different legal policies adopted by different countries concerning electronic payment systems. On average, quite a number of respondents did not have an opinion, that is, they were neutral on whether there are any benefits that come with electronic payment systems.

Table 2: Benefits of EPS to Commercial Banks in Kenya

\begin{tabular}{|c|c|c|c|c|c|c|c|c|c|c|c|c|}
\hline & \multicolumn{2}{|c|}{$\begin{array}{l}\text { Strongly } \\
\text { Disagree }\end{array}$} & \multicolumn{2}{|c|}{ Disagree } & \multicolumn{2}{|c|}{ Neutral } & \multicolumn{2}{|c|}{ Agree } & \multicolumn{2}{|c|}{$\begin{array}{l}\text { Strongly } \\
\text { Agree }\end{array}$} & & \\
\hline & Count & $\mathrm{N} \%$ & Count & $\mathrm{N} \%$ & Count & $\mathrm{N} \%$ & Count & $\mathrm{N} \%$ & Count & $\mathrm{N} \%$ & Count & Table N \% \\
\hline ATM: global reach. & 2 & $3 \%$ & 9 & $14 \%$ & 18 & $27 \%$ & 27 & $41 \%$ & 10 & $15 \%$ & 66 & $100.0 \%$ \\
\hline ATM: high revenue & 6 & $9 \%$ & 9 & $13 \%$ & 17 & $25 \%$ & 31 & $46 \%$ & 5 & $7 \%$ & 68 & $100.0 \%$ \\
\hline ATM: low cost & 3 & $4 \%$ & 5 & $7 \%$ & 35 & $51 \%$ & 18 & $26 \%$ & 7 & $10 \%$ & 68 & $100.0 \%$ \\
\hline ATM privacy features & 4 & $6 \%$ & 9 & $13 \%$ & 13 & $19 \%$ & 37 & $54 \%$ & 5 & $7 \%$ & 68 & $100.0 \%$ \\
\hline ATM: mass customization & 5 & $7 \%$ & 10 & $15 \%$ & 18 & $26 \%$ & 26 & $38 \%$ & 9 & $13 \%$ & 68 & $100.0 \%$ \\
\hline ATM: $24 / 7$ services & 6 & $9 \%$ & 3 & $4 \%$ & 14 & $21 \%$ & 25 & $37 \%$ & 20 & $29 \%$ & 68 & $100.0 \%$ \\
\hline Debit Card: global reach & 3 & $4 \%$ & 9 & $13 \%$ & 28 & $41 \%$ & 19 & $28 \%$ & 9 & $13 \%$ & 68 & $100.0 \%$ \\
\hline Debit Card: high revenue & 2 & $3 \%$ & 10 & $15 \%$ & 30 & $44 \%$ & 20 & $29 \%$ & 6 & $9 \%$ & 68 & $100.0 \%$ \\
\hline Debit Card: Low cost & 3 & $4 \%$ & 9 & $13 \%$ & 28 & $41 \%$ & 23 & $34 \%$ & 5 & $7 \%$ & 68 & $100.0 \%$ \\
\hline $\begin{array}{l}\text { Debit Card: privacy } \\
\text { features }\end{array}$ & 4 & $6 \%$ & 2 & $3 \%$ & 26 & $38 \%$ & 32 & $47 \%$ & 4 & $6 \%$ & 68 & $100.0 \%$ \\
\hline $\begin{array}{l}\text { Debit Card: mass } \\
\text { customization }\end{array}$ & 6 & $9 \%$ & 11 & $16 \%$ & 25 & $37 \%$ & 23 & $34 \%$ & 3 & $4 \%$ & 68 & $100.0 \%$ \\
\hline Debit Card: $24 / 7$ services & 0 & $0 \%$ & 12 & $18 \%$ & 23 & $34 \%$ & 26 & $38 \%$ & 7 & $10 \%$ & 68 & $100.0 \%$ \\
\hline Credit Card: global reach & 1 & $1 \%$ & 13 & $19 \%$ & 27 & $40 \%$ & 17 & $25 \%$ & 10 & $15 \%$ & 68 & $100.0 \%$ \\
\hline Credit Card: high revenue & 7 & $10 \%$ & 4 & $6 \%$ & 25 & $37 \%$ & 26 & $38 \%$ & 6 & $9 \%$ & 68 & $100.0 \%$ \\
\hline Credit Card: Low cost & 9 & $13 \%$ & 1 & $1 \%$ & 34 & $50 \%$ & 21 & $31 \%$ & 3 & $4 \%$ & 68 & $100.0 \%$ \\
\hline $\begin{array}{l}\text { Credit Card: privacy } \\
\text { features }\end{array}$ & 1 & $1 \%$ & 6 & $9 \%$ & 27 & $40 \%$ & 31 & $46 \%$ & 3 & $4 \%$ & 68 & $100.0 \%$ \\
\hline $\begin{array}{l}\text { Credit Card: mass } \\
\text { customization }\end{array}$ & 11 & $16 \%$ & 4 & $6 \%$ & 24 & $35 \%$ & 26 & $38 \%$ & 3 & $4 \%$ & 68 & $100.0 \%$ \\
\hline Credit Card: $24 / 7$ services & 3 & $4 \%$ & 2 & $3 \%$ & 20 & $29 \%$ & 29 & $43 \%$ & 14 & $21 \%$ & 68 & $100.0 \%$ \\
\hline $\begin{array}{l}\text { Mobile Payment: global } \\
\text { reach }\end{array}$ & 4 & $6 \%$ & 13 & $19 \%$ & 19 & $28 \%$ & 26 & $38 \%$ & 6 & $9 \%$ & 68 & $100.0 \%$ \\
\hline
\end{tabular}




\begin{tabular}{|c|c|c|c|c|c|c|c|c|c|c|c|c|}
\hline & \multicolumn{2}{|c|}{$\begin{array}{l}\text { Strongly } \\
\text { Disagree }\end{array}$} & \multicolumn{2}{|c|}{ Disagree } & \multicolumn{2}{|c|}{ Neutral } & \multicolumn{2}{|c|}{ Agree } & \multicolumn{2}{|c|}{$\begin{array}{l}\text { Strongly } \\
\text { Agree }\end{array}$} & & \\
\hline & Count & $\mathrm{N} \%$ & Count & $\mathrm{N} \%$ & Count & $\mathrm{N} \%$ & Count & $\mathrm{N} \%$ & Count & $\mathrm{N} \%$ & Count & Table N \% \\
\hline $\begin{array}{l}\text { Mobile Payment: high } \\
\text { revenue }\end{array}$ & 7 & $10 \%$ & 9 & $13 \%$ & 7 & $10 \%$ & 32 & $47 \%$ & 13 & $19 \%$ & 68 & $100.0 \%$ \\
\hline $\begin{array}{l}\text { Mobile Payment: capital } \\
\text { cost }\end{array}$ & 3 & $4 \%$ & 8 & $12 \%$ & 10 & $15 \%$ & 35 & $51 \%$ & 12 & $18 \%$ & 68 & $100.0 \%$ \\
\hline $\begin{array}{l}\text { Mobile Payment: privacy } \\
\text { features }\end{array}$ & 3 & $4 \%$ & 8 & $12 \%$ & 21 & $31 \%$ & 26 & $38 \%$ & 10 & $15 \%$ & 68 & $100.0 \%$ \\
\hline $\begin{array}{l}\text { Mobile Payment: mass } \\
\text { customization }\end{array}$ & 4 & $6 \%$ & 10 & $15 \%$ & 18 & $26 \%$ & 30 & $44 \%$ & 6 & $9 \%$ & 68 & $100.0 \%$ \\
\hline $\begin{array}{l}\text { Mobile Payment: } 24 / 7 \\
\text { services }\end{array}$ & 3 & $4 \%$ & 6 & $9 \%$ & 13 & $19 \%$ & 28 & $41 \%$ & 18 & $26 \%$ & 68 & $100.0 \%$ \\
\hline $\begin{array}{l}\text { Internet Banking: global } \\
\text { reach }\end{array}$ & 14 & $21 \%$ & 6 & $9 \%$ & 1 & $1 \%$ & 29 & $43 \%$ & 18 & $26 \%$ & 68 & $100.0 \%$ \\
\hline $\begin{array}{l}\text { Internet Banking: high } \\
\text { revenue }\end{array}$ & 4 & $6 \%$ & 10 & $15 \%$ & 20 & $29 \%$ & 24 & $35 \%$ & 10 & $15 \%$ & 68 & $100.0 \%$ \\
\hline $\begin{array}{l}\text { Internet Banking: capital } \\
\text { cost }\end{array}$ & 9 & $13 \%$ & 6 & $9 \%$ & 10 & $15 \%$ & 22 & $32 \%$ & 21 & $31 \%$ & 68 & $100.0 \%$ \\
\hline $\begin{array}{l}\text { Internet Banking: privacy } \\
\text { features }\end{array}$ & 10 & $15 \%$ & 11 & $16 \%$ & 11 & $16 \%$ & 25 & $37 \%$ & 11 & $16 \%$ & 68 & $100.0 \%$ \\
\hline $\begin{array}{l}\text { Internet Banking: mass } \\
\text { customization }\end{array}$ & 5 & $7 \%$ & 12 & $18 \%$ & 16 & $24 \%$ & 21 & $31 \%$ & 14 & $21 \%$ & 68 & $100.0 \%$ \\
\hline $\begin{array}{l}\text { Internet Banking: } 24 / 7 \\
\text { services }\end{array}$ & 4 & $6 \%$ & 6 & $9 \%$ & 10 & $15 \%$ & 27 & $40 \%$ & 21 & $31 \%$ & 68 & $100.0 \%$ \\
\hline
\end{tabular}

\subsection{Challenges Facing Implementation of Electronic Payment Systems}

The challenges facing electronic payment systems in Kenya were recorded as follows in table 4.5 below. There is strong evidence that the challenges actually exist and affect the adoption of electronic payment systems. Online fraud (strongly agree - 16\%) is one of the most challenging factors in adoption of electronic payment systems. The other equally worrying challenges are different software platforms (12\%) and lack of cross border EPS support (14\%). Where different implementation platforms are involved, banks find it difficult to interface with each other hence hampering smooth interbank transactions. Where banks have overseas branches, the challenge of inter-border transaction is also difficult if the host country does not ratify electronic payment as one of the official modes of transaction.

Table 3: Electronic Payment Challenges Facing Banks

\begin{tabular}{|c|c|c|c|c|c|c|c|c|c|c|c|c|}
\hline & \multicolumn{2}{|c|}{ Strongly Disagree } & \multicolumn{2}{|c|}{ Disagree } & \multicolumn{2}{|c|}{ Neutral } & \multicolumn{2}{|c|}{ Agree } & \multicolumn{2}{|c|}{ Strongly Agree } & \multicolumn{2}{|r|}{ Total } \\
\hline & Count & $\mathrm{N} \%$ & Count & $\mathrm{N} \%$ & Count & $\mathrm{N} \%$ & Count & $\mathrm{N} \%$ & Count & $\mathrm{N} \%$ & Count & Total N \% \\
\hline different e-payment platform & 4 & $6 \%$ & 2 & $3 \%$ & 33 & $48 \%$ & 22 & $32 \%$ & 8 & $12 \%$ & 69 & $100 \%$ \\
\hline Low uptake cashless society & 4 & $6 \%$ & 13 & $19 \%$ & 17 & $25 \%$ & 29 & $42 \%$ & 6 & $9 \%$ & 69 & $100 \%$ \\
\hline Increased online fraud & 4 & $6 \%$ & 5 & $7 \%$ & 23 & $33 \%$ & 26 & $38 \%$ & 11 & $16 \%$ & 69 & $100 \%$ \\
\hline
\end{tabular}


ISSN 2278-5612

\begin{tabular}{|c|c|c|c|c|c|c|c|c|c|c|c|c|}
\hline & \multicolumn{2}{|c|}{ Strongly Disagree } & \multicolumn{2}{|c|}{ Disagree } & \multicolumn{2}{|c|}{ Neutral } & \multicolumn{2}{|c|}{ Agree } & \multicolumn{2}{|c|}{ Strongly Agree } & \multicolumn{2}{|c|}{ Total } \\
\hline & Count & $\mathrm{N} \%$ & Count & $N \%$ & Count & $\mathrm{N} \%$ & Count & $N \%$ & Count & $\mathrm{N} \%$ & Count & Total N \% \\
\hline Over reliance on IT & 2 & $3 \%$ & 9 & $13 \%$ & 22 & $32 \%$ & 32 & $46 \%$ & 4 & $6 \%$ & 69 & $100 \%$ \\
\hline fear for layoffs & 8 & $12 \%$ & 12 & $17 \%$ & 19 & $28 \%$ & 26 & $38 \%$ & 4 & $6 \%$ & 69 & $100 \%$ \\
\hline $\begin{array}{l}\text { cross border e-payment } \\
\text { system }\end{array}$ & 2 & $3 \%$ & 13 & $19 \%$ & 22 & $32 \%$ & 22 & $32 \%$ & 10 & $14 \%$ & 69 & $100 \%$ \\
\hline
\end{tabular}

The least challenge came from fear for layoffs (12\% strongly disagree). Where change management is professionally carried out, staffs have no intrinsic fear of future consequences. The findings of the major challenges were presented in a bar chart for clarity of the opinion as shown in Figure 4.1 below. Online fraud bar charts indicate a larger portion of respondents with certain levels of agreement.

\section{Figure 4: Increased Online Fraud Effects on Approval of E-Payment Systems}

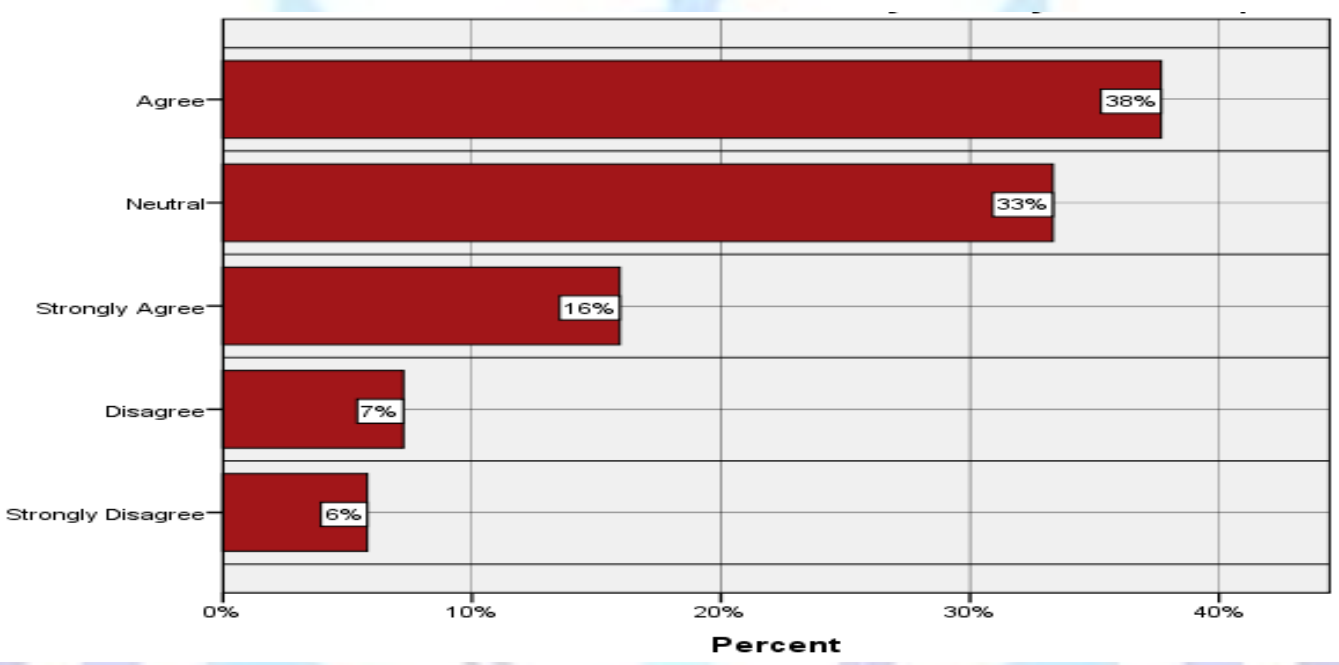

Banks' use of different electronic payment software and platform also has a strong indication for being a stumbling block to success of electronic payment systems deployment by banks.

Lack of cross-border e-payment system legal and technical support proves to be a huge challenge too. In the absence of an ex ante agreed upon resolution and burden-sharing mechanism and deteriorating health of the bank, incentive conflicts escalate and supervisory cooperation breaks down. Those who were neutral tied up at $22 \%$ with agreed respondents. Perhaps any slightest cross border transactions inconvenience would see them join the 'agree' group.

Figure 5: Lack of Cross-Border E-payment Systems

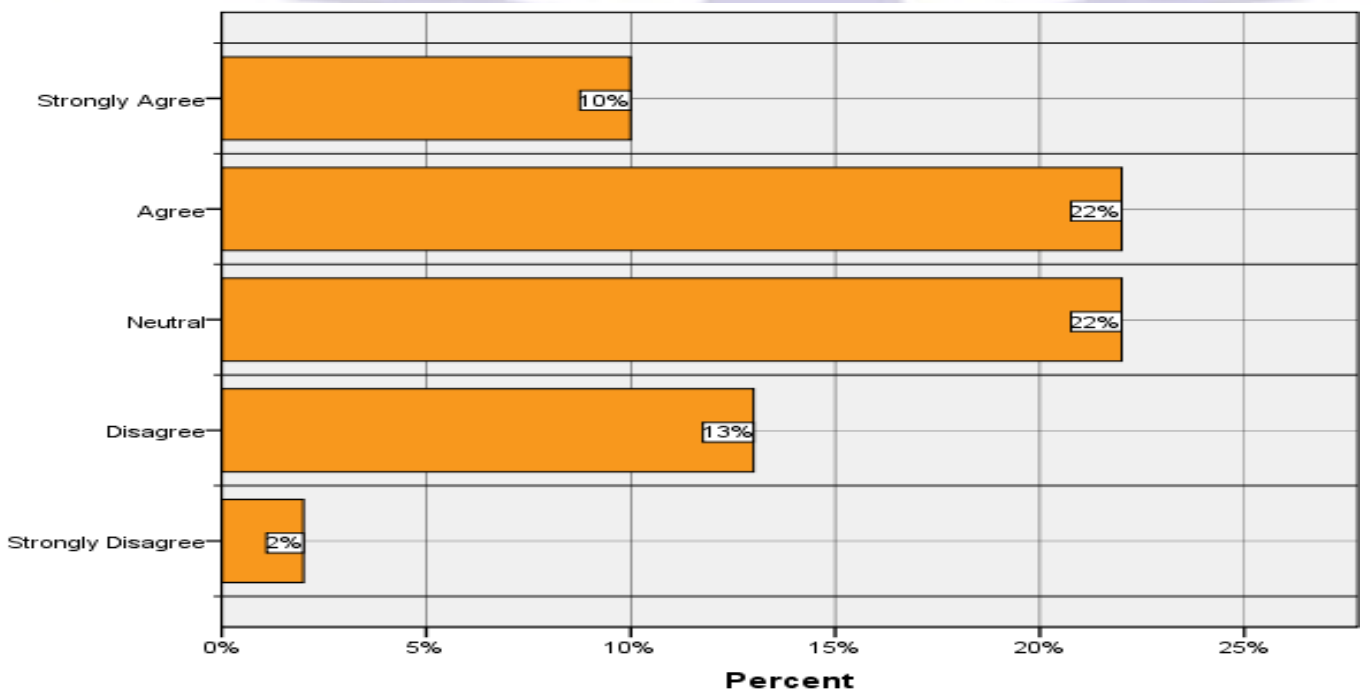




\subsection{Key Drivers of Electronic Payment Systems}

The research data obtained through questionnaires from banks respondents touching on key drivers for adoption of electronic payment systems are illustrated in the table 4 below. The answers are divided into two main categories of Yes and No for each payment method against the rows which constitute the key drivers for adoption. Majority of the respondents approved the role of drivers in adoption of electronic payment systems. Mobile phone payment system led the pack with $85 \%$ suggesting that they may take shorter time to transact. Improved system security providing trust and confidence to banks deployment of ATMs scored the highest probability of $79 \%$. Perceived usefulness of ATMs probability stood at $81 \%$ while banks capacity to deploy and manage credit cards electronic systems probability was $81 \%$. In contrast to the above supportive indicators, $47 \%$ mobile and internet method of payments was considered to have higher transaction risks. The opinion could be shaped by the fact that once the password is known to a third party, fraud can take place undetectably.

Table 4: The Main Drivers of Electronic Payment Systems

\begin{tabular}{|l|r|r|r|r|r|r|r|r|r|r|}
\hline & \multicolumn{5}{|c|}{ Yes } & \multicolumn{3}{|c|}{ No } \\
\cline { 2 - 11 } & ATM & $\begin{array}{l}\text { Debit } \\
\text { Card }\end{array}$ & $\begin{array}{l}\text { Credit } \\
\text { Card }\end{array}$ & $\begin{array}{l}\text { Mobile } \\
\text { Payment }\end{array}$ & $\begin{array}{l}\text { Internet } \\
\text { Payment }\end{array}$ & ATM & $\begin{array}{l}\text { Debit } \\
\text { Card }\end{array}$ & $\begin{array}{l}\text { Credit } \\
\text { Card }\end{array}$ & $\begin{array}{l}\text { Mobile } \\
\text { Payment }\end{array}$ & $\begin{array}{l}\text { Internet } \\
\text { Payment }\end{array}$ \\
\hline $\begin{array}{l}\text { Improved e- } \\
\text { system security }\end{array}$ & $79 \%$ & $54 \%$ & $59 \%$ & $75 \%$ & $59 \%$ & $21 \%$ & $46 \%$ & $41 \%$ & $25 \%$ & $41 \%$ \\
\hline User anonymity & $66 \%$ & $66 \%$ & $65 \%$ & $62 \%$ & $68 \%$ & $34 \%$ & $34 \%$ & $35 \%$ & $38 \%$ & $32 \%$ \\
\hline $\begin{array}{l}\text { E-payment lon } \\
\text { term strategy }\end{array}$ & $71 \%$ & $65 \%$ & $69 \%$ & $78 \%$ & $77 \%$ & $29 \%$ & $35 \%$ & $31 \%$ & $22 \%$ & $23 \%$ \\
\hline $\begin{array}{l}\text { Low costs of } \\
\text { implementation }\end{array}$ & $62 \%$ & $62 \%$ & $54 \%$ & $70 \%$ & $65 \%$ & $38 \%$ & $38 \%$ & $46 \%$ & $30 \%$ & $35 \%$ \\
\hline $\begin{array}{l}\text { Perceived } \\
\text { usefulness }\end{array}$ & $82 \%$ & $68 \%$ & $73 \%$ & $75 \%$ & $65 \%$ & $18 \%$ & $32 \%$ & $27 \%$ & $25 \%$ & $35 \%$ \\
\hline $\begin{array}{l}\text { Low transaction } \\
\text { risk }\end{array}$ & $67 \%$ & $65 \%$ & $55 \%$ & $53 \%$ & $53 \%$ & $33 \%$ & $35 \%$ & $45 \%$ & $47 \%$ & $47 \%$ \\
\hline System capacity & $77 \%$ & $76 \%$ & $81 \%$ & $66 \%$ & $77 \%$ & $23 \%$ & $24 \%$ & $19 \%$ & $34 \%$ & $23 \%$ \\
\hline $\begin{array}{l}\text { Short transaction } \\
\text { time }\end{array}$ & $70 \%$ & $84 \%$ & $75 \%$ & $85 \%$ & $67 \%$ & $30 \%$ & $16 \%$ & $25 \%$ & $15 \%$ & $33 \%$ \\
\hline $\begin{array}{l}\text { System } \\
\text { opportunities }\end{array}$ & $74 \%$ & $62 \%$ & $69 \%$ & $75 \%$ & $80 \%$ & $26 \%$ & $38 \%$ & $31 \%$ & $25 \%$ & $20 \%$ \\
\hline $\begin{array}{l}\text { Changes in } \\
\text { lifestyle }\end{array}$ & $72 \%$ & $69 \%$ & $73 \%$ & $82 \%$ & $83 \%$ & $28 \%$ & $31 \%$ & $27 \%$ & $18 \%$ & $17 \%$ \\
\hline
\end{tabular}

There is generally high score in changes of lifestyle having effect on adoption of EPS. Internet method (80\%) provides the highest chances of creating more opportunities than all the other EPS.

\subsection{Correlation and Regression of Determinants of E-Payment Systems}

The technique of correlation is used to test the statistical significance of the association. On the other hand, regression analysis is used to describe the relationship precisely by means of an equation that has predictive value. The two analyses techniques are important because of their different roles.

\subsubsection{Correlation of Determinants of EPS}

Pearson correlation is used to evaluate the relationship between variables and its matrix is an important indicator that tests the linear relationship, between the variables. The matrix also helps to determine the strength of the variables in the model, that is, which variable best explains the relationship between determinants of e-payment systems and the current method of electronic payment systems used by commercial banks in Kenya. This is important since it helps in deciding which variable(s) to drop from the equation. Table 5 presents the correlation matrix in levels. Table 5 also shows that there is no statistical relationship between key EPS determinants with the use of ATM as an electronic 
payment method. However, improved systems security, perceived usefulness, low transaction risk, capacity of banks system and the presence of bank branches had a positive relationship with use of ATM method.

Table 5: Pearson Correlations - ATM Payment Method

\begin{tabular}{|c|c|c|c|c|c|c|c|c|c|}
\hline & & 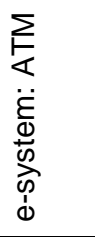 & 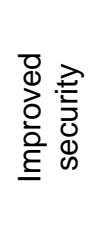 & 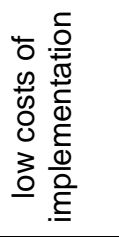 & 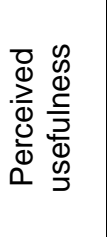 & 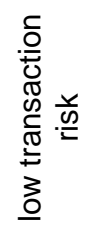 & 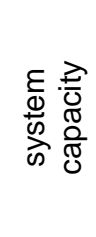 & 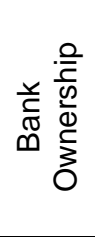 & 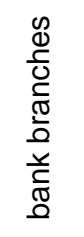 \\
\hline \multirow{3}{*}{ e-system: ATM } & Pearson Correlation & 1 & .089 & .194 & -.144 & .229 & .076 & $-.252^{*}$ & $.265^{*}$ \\
\hline & Sig. (2-tailed) & & .465 & .125 & .241 & .071 & .530 & .043 & .032 \\
\hline & $\mathrm{N}$ & 70 & 70 & 64 & 68 & 63 & 70 & 65 & 66 \\
\hline \multirow{3}{*}{$\begin{array}{l}\text { Improved } \\
\text { security }\end{array}$} & Pearson Correlation & .089 & 1 & .010 & -.060 & .222 & $-.284^{*}$ & .046 & .214 \\
\hline & Sig. (2-tailed) & .465 & & .937 & .626 & .081 & .017 & .715 & .084 \\
\hline & $\mathrm{N}$ & 70 & 70 & 64 & 68 & 63 & 70 & 65 & 66 \\
\hline \multirow{3}{*}{$\begin{array}{l}\text { low costs of } \\
\text { implementation }\end{array}$} & Pearson Correlation & .194 & .010 & 1 & -.078 & .193 & -.224 & .064 & .105 \\
\hline & Sig. (2-tailed) & .125 & .937 & & .545 & .146 & .076 & .632 & .415 \\
\hline & $\mathrm{N}$ & 64 & 64 & 64 & 62 & 58 & 64 & 59 & 62 \\
\hline \multirow{3}{*}{$\begin{array}{l}\text { Perceived } \\
\text { usefulness }\end{array}$} & Pearson Correlation & -.144 & -.060 & -.078 & 1 & $-.257^{*}$ & .107 & .023 & .038 \\
\hline & Sig. (2-tailed) & .241 & .626 & .545 & & .042 & .385 & .857 & .764 \\
\hline & $\mathrm{N}$ & 68 & 68 & 62 & 68 & 63 & 68 & 63 & 64 \\
\hline \multirow{3}{*}{$\begin{array}{l}\text { low transaction } \\
\text { risk }\end{array}$} & Pearson Correlation & .229 & .222 & .193 & $-.257^{*}$ & 1 & -.216 & -.098 & -.220 \\
\hline & Sig. (2-tailed) & .071 & .081 & .146 & .042 & & .089 & .466 & .094 \\
\hline & $N$ & 63 & 63 & 58 & 63 & 63 & 63 & 58 & 59 \\
\hline \multirow{3}{*}{$\begin{array}{l}\text { Banks system } \\
\text { capacity }\end{array}$} & Pearson Correlation & .076 & $-.284^{*}$ & -.224 & .107 & -.216 & 1 & -.101 & -.105 \\
\hline & Sig. (2-tailed) & .530 & .017 & .076 & .385 & .089 & & .423 & .400 \\
\hline & $N$ & 70 & 70 & 64 & 68 & 63 & 70 & 65 & 66 \\
\hline \multirow{3}{*}{$\begin{array}{l}\text { Bank } \\
\text { Ownership }\end{array}$} & Pearson Correlation & $-.252^{*}$ & .046 & .064 & .023 & -.098 & -.101 & 1 & -.009 \\
\hline & Sig. (2-tailed) & .043 & .715 & .632 & .857 & .466 & .423 & & .943 \\
\hline & $\mathrm{N}$ & 65 & 65 & 59 & 63 & 58 & 65 & 65 & 63 \\
\hline \multirow{3}{*}{ Bank branches } & Pearson Correlation & $.265^{*}$ & .214 & .105 & .038 & -.220 & -.105 & -.009 & 1 \\
\hline & Sig. (2-tailed) & .032 & .084 & .415 & .764 & .094 & .400 & .943 & \\
\hline & $\mathrm{N}$ & 66 & 66 & 62 & 64 & 59 & 66 & 63 & 66 \\
\hline
\end{tabular}

${ }^{*}$. Correlation is significant at the 0.05 level (2-tailed). 
The number of bank's branches indicates a strong positive relationship with use of credit cards as shown in table 6 below.

Table 6: Pearson Correlation - Credit Card Payment Method

\begin{tabular}{|c|c|c|c|c|c|c|c|c|c|}
\hline & & 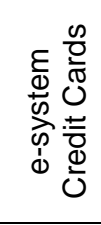 & 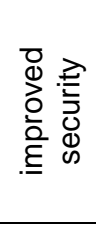 & 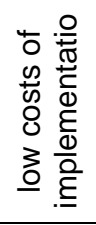 & 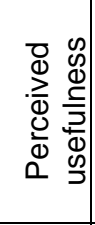 & 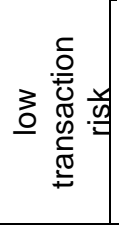 & 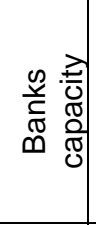 & 号 & 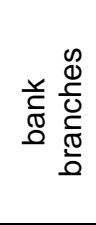 \\
\hline \multirow{3}{*}{$\begin{array}{l}\text { e-system Credit } \\
\text { Cards }\end{array}$} & Pearson Correlation & 1 & $.295^{*}$ & $.311^{*}$ & .017 & $-.295^{*}$ & .097 & -.127 & $.615^{* *}$ \\
\hline & Sig. (2-tailed) & & .018 & .019 & .900 & .020 & .449 & .336 & .000 \\
\hline & $N$ & 64 & 64 & 57 & 59 & 62 & 63 & 59 & 60 \\
\hline \multirow{3}{*}{$\begin{array}{l}\text { improved } \\
\text { security }\end{array}$} & Pearson Correlation & $.295^{*}$ & 1 & .070 & $.392^{*}$ & -.044 & .120 & -.174 & $.298^{*}$ \\
\hline & Sig. (2-tailed) & .018 & & .596 & .001 & .733 & .334 & .172 & .017 \\
\hline & $N$ & 64 & 68 & 59 & 63 & 64 & 67 & 63 & 64 \\
\hline \multirow{3}{*}{$\begin{array}{l}\text { low costs of } \\
\text { implementation }\end{array}$} & Pearson Correlation & $.311^{\star}$ & .070 & 1 & $.317^{\star}$ & .028 & .161 & -.100 & .120 \\
\hline & Sig. (2-tailed) & .019 & .596 & & .015 & .836 & .226 & .462 & .384 \\
\hline & $N$ & 57 & 59 & 59 & 58 & 56 & 58 & 56 & 55 \\
\hline \multirow{3}{*}{$\begin{array}{l}\text { Perceived } \\
\text { usefulness }\end{array}$} & Pearson Correlation & .017 & $.392^{* \star}$ & $.317^{*}$ & 1 & $.264^{*}$ & .240 & -.019 & $.316^{*}$ \\
\hline & Sig. (2-tailed) & .900 & .001 & .015 & & .043 & .061 & .884 & .015 \\
\hline & $N$ & 59 & 63 & 58 & 63 & 59 & 62 & 60 & 59 \\
\hline \multirow{3}{*}{$\begin{array}{l}\text { low transaction } \\
\text { risk }\end{array}$} & Pearson Correlation & $-.295^{*}$ & -.044 & .028 & $.264^{*}$ & 1 & .223 & -.249 & -.112 \\
\hline & Sig. (2-tailed) & .020 & .733 & .836 & .043 & & .078 & .057 & .396 \\
\hline & $N$ & 62 & 64 & 56 & 59 & 64 & 63 & 59 & 60 \\
\hline \multirow{3}{*}{ Banks capacity } & Pearson Correlation & .097 & .120 & .161 & .240 & .223 & 1 & $-.250^{*}$ & -.137 \\
\hline & Sig. (2-tailed) & .449 & .334 & .226 & .061 & .078 & & .048 & .281 \\
\hline & $N$ & 63 & 67 & 58 & 62 & 63 & 67 & 63 & 64 \\
\hline \multirow{3}{*}{$\begin{array}{l}\text { Bank } \\
\text { Ownership }\end{array}$} & Pearson Correlation & -.127 & -.174 & -.100 & .019 & -.249 & $.250^{-}$ & 1 & -.009 \\
\hline & Sig. (2-tailed) & .336 & .172 & .462 & .884 & .057 & .048 & & .943 \\
\hline & $N$ & 59 & 63 & 56 & 60 & 59 & 63 & 65 & 63 \\
\hline \multirow{2}{*}{ bank branches } & Pearson Correlation & $.615^{\star *}$ & $.298^{*}$ & .120 & $.316^{*}$ & -.112 & .137 & -.009 & 1 \\
\hline & Sig. (2-tailed) & .000 & .017 & .384 & .015 & .396 & .281 & .943 & \\
\hline
\end{tabular}




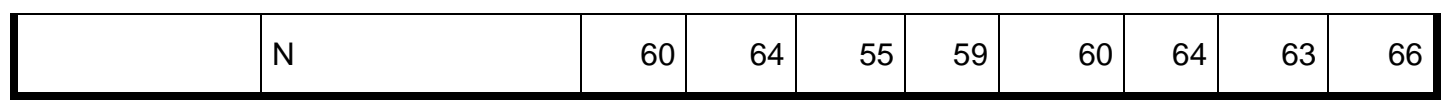

*. Correlation is significant at the 0.05 level (2-tailed).

**. Correlation is significant at the 0.01 level (2-tailed).

A similar relationship is also exhibited in table 7 below where internet payment method has a positive relationship with presence of bank branches.

Table 7: Pearson Correlation - Internet Payment Method

\begin{tabular}{|c|c|c|c|c|c|c|c|c|c|}
\hline & & 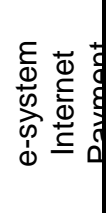 & 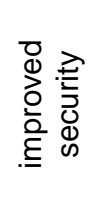 & 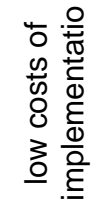 & 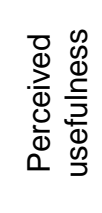 & 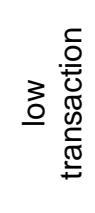 & 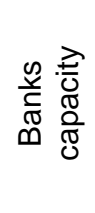 & 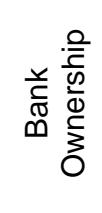 & 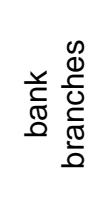 \\
\hline & Pearson Correlation & 1 & -.055 & -.060 & .007 & -.143 & .138 & .181 & $.377^{\star *}$ \\
\hline \multirow{3}{*}{$\begin{array}{l}\text { e-system } \\
\text { Internet } \\
\text { Payment }\end{array}$} & Sig. (2-tailed) & & .671 & .650 & .957 & .275 & .280 & .163 & .003 \\
\hline & $\mathrm{N}$ & 63 & 63 & 59 & 58 & 60 & 63 & 61 & 61 \\
\hline & Pearson Correlation & -.055 & 1 & -.005 & $.371^{* *}$ & $.359^{* *}$ & -.043 & -.173 & .035 \\
\hline \multirow[t]{3}{*}{$\begin{array}{l}\text { improved } \\
\text { security }\end{array}$} & Sig. (2-tailed) & .671 & & .967 & .002 & .003 & .721 & .168 & .779 \\
\hline & $\mathrm{N}$ & 63 & 70 & 63 & 65 & 66 & 70 & 65 & 66 \\
\hline & Pearson Correlation & -.060 & -.005 & 1 & $.269^{*}$ & .024 & $.329^{\star *}$ & -.062 & .051 \\
\hline \multirow[t]{3}{*}{$\begin{array}{l}\text { low costs of } \\
\text { implementation }\end{array}$} & Sig. (2-tailed) & .650 & .967 & & .038 & .858 & .008 & .644 & .700 \\
\hline & $\mathrm{N}$ & 59 & 63 & 63 & 60 & 60 & 63 & 58 & 59 \\
\hline & Pearson Correlation & .007 & $.371^{* *}$ & $.269^{*}$ & 1 & .149 & .160 & $-.251^{*}$ & .088 \\
\hline \multirow{3}{*}{$\begin{array}{l}\text { Perceived } \\
\text { usefulness }\end{array}$} & Sig. (2-tailed) & .957 & .002 & .038 & & .252 & .203 & .049 & .499 \\
\hline & $\mathrm{N}$ & 58 & 65 & 60 & 65 & 61 & 65 & 62 & 61 \\
\hline & Pearson Correlation & -.143 & $.359^{* *}$ & .024 & .149 & 1 & .106 & -.213 & -.132 \\
\hline \multirow[t]{3}{*}{$\begin{array}{l}\text { low transaction } \\
\text { risk }\end{array}$} & Sig. (2-tailed) & .275 & .003 & .858 & .252 & & .398 & .099 & .305 \\
\hline & $\mathrm{N}$ & 60 & 66 & 60 & 61 & 66 & 66 & 61 & 62 \\
\hline & Pearson Correlation & .138 & -.043 & $.329^{* *}$ & .160 & .106 & 1 & -.134 & -.125 \\
\hline \multirow[t]{3}{*}{ Banks capacity } & Sig. (2-tailed) & .280 & .721 & .008 & .203 & .398 & & .287 & .316 \\
\hline & $\mathrm{N}$ & 63 & 70 & 63 & 65 & 66 & 70 & 65 & 66 \\
\hline & Pearson Correlation & .181 & -.173 & -.062 & $-.251^{*}$ & -.213 & -.134 & 1 & -.009 \\
\hline \multirow[t]{2}{*}{$\begin{array}{l}\text { Bank } \\
\text { Ownership }\end{array}$} & Sig. (2-tailed) & .163 & .168 & .644 & .049 & .099 & .287 & & .943 \\
\hline & $\mathrm{N}$ & 61 & 65 & 58 & 62 & 61 & 65 & 65 & 63 \\
\hline bank branches & Pearson Correlation & $.377^{\star *}$ & .035 & .051 & .088 & -.132 & -.125 & -.009 & 1 \\
\hline
\end{tabular}


Table 7: Pearson Correlation - Internet Payment Method

\begin{tabular}{|c|c|c|c|c|c|c|c|c|}
\hline & 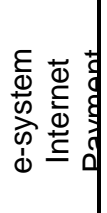 & 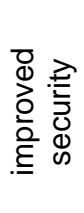 & 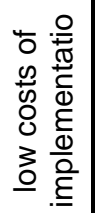 & 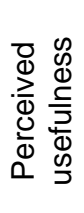 & 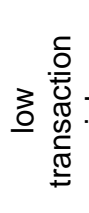 & 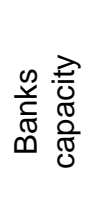 & 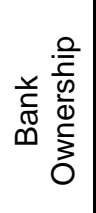 & 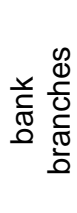 \\
\hline Sig. (2-tailed) & .003 & .779 & .700 & .499 & .305 & .316 & .943 & \\
\hline $\mathrm{N}$ & 61 & 66 & 59 & 61 & 62 & 66 & 63 & 66 \\
\hline
\end{tabular}

${ }^{\star *}$. Correlation is significant at the 0.01 level (2-tailed).

*. Correlation is significant at the 0.05 level (2-tailed).

\subsubsection{Determinants of E-payment Systems Regression}

The five factors of research framework are improved systems security, perceived bank trust/ low risk, enhanced infrastructure capacity, cost reduction and perceived benefits. To test for statistical significance, these factors were regressed against the identified methods of electronic payments by commercial banks in Kenya. Regression was used since it is the best technique used to measure the effects of two or more independent variables on a single dependent variable measured on interval or ratio scales (Walliman, 2011).

A regression of ATM as an EPS is shown in table 4.7 below. Only $12.6 \%$ of variables in the use of ATM payment method can be explained by all the independent variables.

Table 8: ATM Payment Regression

\section{Model Summary}

\begin{tabular}{|l|r|r|r|r|}
\hline Model & $\mathrm{R}$ & R Square & \multicolumn{1}{|c|}{$\begin{array}{c}\text { Adjusted R } \\
\text { Square }\end{array}$} & $\begin{array}{l}\text { Std. Error of the } \\
\text { Estimate }\end{array}$ \\
\hline 1 & $.355^{\mathrm{a}}$ & .126 & .042 & .301 \\
\hline
\end{tabular}

a. Predictors: (Constant), ATM: Perceived usefulness, ATM: low costs of implementation, ATM: Improved e-system security, ATM: system capacity, ATM: low transaction risk

The table above indicates that $12.6 \%$ of total ATM usage variance is explained by all the independent variables. The remaining $87.4 \%$ is explained by other factors unknown to the researcher.

ANOVA $^{\mathrm{a}}$

\begin{tabular}{|c|c|c|c|c|c|c|}
\hline \multicolumn{2}{|c|}{ Model } & Sum of Squares & df & Mean Square & $F$ & Sig. \\
\hline \multirow{3}{*}{1} & Regression & .679 & 5 & .136 & 1.503 & $.205^{\mathrm{b}}$ \\
\hline & Residual & 4.700 & 52 & .090 & & \\
\hline & Total & 5.379 & 57 & & & \\
\hline
\end{tabular}

a. Dependent Variable: Bank e-system ATM

b. Predictors: (Constant), ATM: Perceived usefulness, ATM: low costs of implementation, ATM: Improved e-system security, ATM: system capacity, ATM: low transaction risk 
Significance of 0.205 implies that there are some differences between variables explaining usage of ATM payment method. Therefore, the hypothesis that drivers of e-payment systems have no effect on the usage of ATM services is rejected since $\mathrm{p}$-value of the $\mathrm{F}$ statistics is $<1$.

\section{Co-efficients ${ }^{\mathrm{a}}$}

\begin{tabular}{|c|c|c|c|c|c|}
\hline \multirow[t]{2}{*}{ Model } & \multicolumn{2}{|c|}{ Unstandardized Coefficients } & \multirow{2}{*}{$\begin{array}{c}\begin{array}{c}\text { Standardized } \\
\text { Coefficients }\end{array} \\
\text { Beta }\end{array}$} & \multirow[t]{2}{*}{$\mathrm{t}$} & \multirow[t]{2}{*}{ Sig. } \\
\hline & B & Std. Error & & & \\
\hline (Constant) & .661 & .186 & & 3.555 & .001 \\
\hline ATM: Improved e-system security & .055 & .111 & .071 & .493 & .624 \\
\hline ATM: low transaction risk & .147 & .093 & .223 & 1.572 & .122 \\
\hline ATM: system capacity & .137 & .099 & .193 & 1.386 & .172 \\
\hline ATM: low costs of implementation & .100 & .084 & .161 & 1.191 & .239 \\
\hline ATM: Perceived usefulness & -.088 & .109 & -.109 & -.805 & .425 \\
\hline
\end{tabular}

a. Dependent Variable: Bank e-system ATM

However, it is important to note that 'low transaction risk' variable increase the usage of ATM payment method by $22 \%(t=1.572)$. Low transaction risk $t$-value is the only one amongst the rest which is closer to the critical value of $95 \%$ confidence interval $(Z$-score $=1.96)$. Perhaps the withdrawal put on ATMs could explain why banks were more confident hence strong relationship.

\section{Table 9: Credit Card Regression}

\section{Model Summary}

\begin{tabular}{|l|r|r|r|r|}
\hline Model & \multicolumn{1}{|c|}{$\mathrm{R}$} & R Square & \multicolumn{1}{|c|}{$\begin{array}{c}\text { Adjusted R } \\
\text { Square }\end{array}$} & $\begin{array}{l}\text { Std. Error of the } \\
\text { Estimate }\end{array}$ \\
\hline 1 & $.678^{\mathrm{a}}$ & .460 & .404 & .342 \\
\hline
\end{tabular}

a. Predictors: (Constant), Credit Card: Banks capacity, Credit Card: low costs of implementation, Credit Card: improved E-payment security, Credit Card: low transaction risk, Credit Card: Perceived usefulness

With credit card payment method, $46 \%$ of Credit card usage is explained by all independent variables in the study. This falls short of the $50 \%$ mark by only $4 \%$.

\section{ANOVA $^{\mathrm{a}}$}

\begin{tabular}{|c|c|c|c|c|c|c|}
\hline \multicolumn{2}{|c|}{ Model } & Sum of Squares & df & Mean Square & $\mathrm{F}$ & Sig. \\
\hline \multirow{3}{*}{1} & Regression & 4.771 & 5 & .954 & 8.179 & $.000^{\mathrm{b}}$ \\
\hline & Residual & 5.600 & 48 & .117 & & \\
\hline & Total & 10.370 & 53 & & & \\
\hline
\end{tabular}

a. Dependent Variable: Bank e-system Credit Cards 
ANOVA $^{\mathrm{a}}$

\begin{tabular}{|l|l|l|l|l|l|}
\hline Model & Sum of Squares & df & Mean Square & F & Sig. \\
\hline
\end{tabular}

b. Predictors: (Constant), Credit Card: Banks capacity, Credit Card: low costs of implementation, Credit Card: improved E-payment security, Credit Card: low transaction risk, Credit Card:

Perceived usefulness

Since the $p$-value of factor statistics is $<1$, there is strong statistical significance between usage of credit card payment method and the drivers of EPS under study. The f-value is 8.179 which is a strong indicator of variables roles in credit card usage.

\section{Co-efficients ${ }^{\mathrm{a}}$}

\begin{tabular}{|c|c|c|c|c|c|}
\hline \multirow[t]{2}{*}{ Model } & \multicolumn{2}{|c|}{ Unstandardized Coefficients } & \multirow{2}{*}{$\begin{array}{c}\begin{array}{c}\text { Standardized } \\
\text { Coefficients }\end{array} \\
\text { Beta }\end{array}$} & \multirow[t]{2}{*}{$\mathrm{t}$} & \multirow[t]{2}{*}{ Sig. } \\
\hline & $\mathrm{B}$ & Std. Error & & & \\
\hline (Constant) & .683 & .123 & & 5.575 & .000 \\
\hline $\begin{array}{l}\text { Credit Card: improved E- } \\
\text { payment security }\end{array}$ & .414 & .105 & .469 & 3.944 & .000 \\
\hline $\begin{array}{l}\text { Credit Card: low costs of } \\
\text { implementation }\end{array}$ & .317 & .101 & .362 & 3.137 & .003 \\
\hline $\begin{array}{l}\text { Credit Card: Perceived } \\
\text { usefulness }\end{array}$ & -.231 & .131 & -.231 & -1.771 & .083 \\
\hline $\begin{array}{l}\text { Credit Card: low transaction } \\
\text { risk }\end{array}$ & -.367 & .099 & -.414 & -3.720 & .001 \\
\hline Credit Card: Banks capacity & .055 & .134 & .049 & .412 & .682 \\
\hline
\end{tabular}

a. Dependent Variable: Bank e-system Credit Cards

The t-test shows three research factors have a strong probability relation with usage of credit cards. These are improved security with probability of $47 \%(\mathrm{t}=3.944)$, low costs of implementation with $36 \%(\mathrm{t}=3.137)$ and low transaction risk with $41 \%(\mathrm{t}=3.720)$. Since ATM cards at times are used as credit cards, it is possible that the security of maximum transaction cap with presence of regional offices helping out customers could explain the positive relation. However, low transaction risk reverses the strong positivity of the first two factors. The banks uneasiness with credit card risk level could be because many vendors participating in online transactions tend to store customers data and that would jeopardise privacy of data policy.

The remaining research variables under study, that is, debit cards, mobile payment and internet banking regression was somehow weak. The f-test in all of them showed slight statistical significance. For the t-tests, banks infrastructure capacity to handle transactions electronically remained positive and strong amongst in all the studied variables. The usage of the variables were explained by $28 \%(t=1.615), 29 \%(t=2.009)$ and $33 \%(t=2.247)$ respectively. More details can be found in table 10,11 and 12 .

Table 10: Debit Card Regression

Model Summary

\begin{tabular}{|l|r|r|r|r|}
\hline Model & \multicolumn{1}{|c|}{$\mathrm{R}$} & R Square & \multicolumn{1}{|c|}{$\begin{array}{c}\text { Adjusted R } \\
\text { Square }\end{array}$} & $\begin{array}{l}\text { Std. Error of the } \\
\text { Estimate }\end{array}$ \\
\hline 1 & $.329^{\mathrm{a}}$ & .108 & .016 & .492 \\
\hline
\end{tabular}


a. Predictors: (Constant), Debit Card: system capacity, Debit Card: low costs of implementation, Debit Card: Perceived usefulness, Debit Card: low risk of transaction, Debit Card: improved E-payment security

ANOVA $^{\mathrm{a}}$

\begin{tabular}{|rr|r|r|r|r|r|}
\hline \multicolumn{1}{|l|}{ Model } & Sum of Squares & df & Mean Square & F & Sig. \\
\hline & Regression & 1.414 & 5 & .283 & 1.168 & $.339^{\mathrm{b}}$ \\
1 & Residual & 11.623 & 48 & .242 & & \\
& 13.037 & 53 & & & \\
\hline
\end{tabular}

a. Dependent Variable: Bank e-system debit Cards

b. Predictors: (Constant), Debit Card: system capacity, Debit Card: low costs of implementation, Debit Card: Perceived usefulness, Debit Card: low risk of transaction, Debit Card: improved Epayment security

\section{Coefficients $^{\mathrm{a}}$}

\begin{tabular}{|c|c|c|c|c|c|c|}
\hline \multicolumn{2}{|c|}{ Model } & \multicolumn{2}{|c|}{ Unstandardized Coefficients } & \multirow{2}{*}{$\begin{array}{c}\begin{array}{c}\text { Standardized } \\
\text { Coefficients }\end{array} \\
\text { Beta }\end{array}$} & \multirow[t]{2}{*}{$\mathrm{t}$} & \multirow[t]{2}{*}{ Sig. } \\
\hline & & B & Std. Error & & & \\
\hline \multirow{6}{*}{1} & (Constant) & .415 & .181 & & 2.290 & .026 \\
\hline & $\begin{array}{l}\text { Debit Card: improved E- } \\
\text { payment security }\end{array}$ & .077 & .159 & .078 & .485 & .630 \\
\hline & $\begin{array}{l}\text { Debit Card: low costs of } \\
\text { implementation }\end{array}$ & -.160 & .140 & -.159 & -1.141 & .259 \\
\hline & $\begin{array}{l}\text { Debit Card: Perceived } \\
\text { usefulness }\end{array}$ & -.043 & .163 & -.041 & -.264 & .793 \\
\hline & $\begin{array}{l}\text { Debit Card: low risk of } \\
\text { transaction }\end{array}$ & .015 & .155 & .015 & .098 & .923 \\
\hline & Debit Card: system capacity & .325 & .201 & .275 & 1.615 & .113 \\
\hline
\end{tabular}

a. Dependent Variable: Bank e-system debit Cards

\section{Table 11: Mobile Payment Regression}

Model Summary

\begin{tabular}{|l|r|r|r|r|}
\hline Model & R & R Square & \multicolumn{1}{|c|}{$\begin{array}{c}\text { Adjusted R } \\
\text { Square }\end{array}$} & $\begin{array}{c}\text { Std. Error of the } \\
\text { Estimate }\end{array}$ \\
\hline 1 & $.411^{\mathrm{a}}$ & .169 & .072 & .295 \\
\hline
\end{tabular}


a. Predictors: (Constant), Mobile Payment: Banks capacity, Mobile Payment: improved E-payment, Mobile Payment: low costs of implementation, Mobile Payment: low risk of transaction, Mobile Payment: Perceived usefulness

ANOVA $^{\mathrm{a}}$

\begin{tabular}{|c|c|c|c|c|c|c|}
\hline \multicolumn{2}{|c|}{ Model } & Sum of Squares & $d f$ & Mean Square & $\mathrm{F}$ & Sig. \\
\hline \multirow{3}{*}{1} & Regression & .758 & 5 & .152 & 1.746 & $.145^{b}$ \\
\hline & Residual & 3.732 & 43 & .087 & & \\
\hline & Total & 4.490 & 48 & & & \\
\hline
\end{tabular}

a. Dependent Variable: Bank e-system Mobile Payment

b. Predictors: (Constant), Mobile Payment: Banks capacity, Mobile Payment: improved Epayment, Mobile Payment: low costs of implementation, Mobile Payment: low risk of transaction, Mobile Payment: Perceived usefulness

\section{Co-efficients $^{a}$}

\begin{tabular}{|c|c|c|c|c|c|c|}
\hline \multirow{2}{*}{\multicolumn{2}{|c|}{ Model }} & \multicolumn{2}{|c|}{ Unstandardized Coefficients } & \multirow{2}{*}{$\begin{array}{c}\begin{array}{c}\text { Standardized } \\
\text { Coefficients }\end{array} \\
\text { Beta }\end{array}$} & \multirow[t]{2}{*}{$\mathrm{t}$} & \multirow[t]{2}{*}{ Sig. } \\
\hline & & B & Std. Error & & & \\
\hline \multirow{6}{*}{1} & (Constant) & .948 & .126 & & 7.535 & .000 \\
\hline & $\begin{array}{l}\text { Mobile Payment: improved } \\
\text { E-payment }\end{array}$ & .051 & .109 & .068 & .468 & .642 \\
\hline & $\begin{array}{l}\text { Mobile Payment: low costs } \\
\text { of implementation }\end{array}$ & -.102 & .102 & -.157 & -.995 & .325 \\
\hline & $\begin{array}{l}\text { Mobile Payment: Perceived } \\
\text { usefulness }\end{array}$ & -.124 & .119 & -.181 & -1.041 & .304 \\
\hline & $\begin{array}{l}\text { Mobile Payment: low risk of } \\
\text { transaction }\end{array}$ & -.116 & .088 & -.192 & -1.325 & .192 \\
\hline & $\begin{array}{l}\text { Mobile Payment: Banks } \\
\text { capacity }\end{array}$ & .190 & .095 & .295 & 2.009 & .051 \\
\hline
\end{tabular}

a. Dependent Variable: Bank e-system Mobile Payment

Table 12: Internet Payment Regression

Model Summary

\begin{tabular}{|l|r|r|r|r|}
\hline Model & $\mathrm{R}$ & R Square & \multicolumn{1}{|c|}{$\begin{array}{c}\text { Adjusted R } \\
\text { Square }\end{array}$} & $\begin{array}{l}\text { Std. Error of the } \\
\text { Estimate }\end{array}$ \\
\hline 1 & $.341^{\mathrm{a}}$ & .116 & .022 & .450 \\
\hline
\end{tabular}


a. Predictors: (Constant), Internet Banking: Banks capacity, Internet Banking: improved E-payment security, Internet Banking: low costs of implementation, Internet Banking: low transaction risk, Internet

Banking: Perceived usefulness

ANOVA $^{\mathrm{a}}$

\begin{tabular}{|c|c|c|c|c|c|c|}
\hline \multicolumn{2}{|c|}{ Model } & Sum of Squares & $d f$ & Mean Square & $\mathrm{F}$ & Sig. \\
\hline \multirow{3}{*}{1} & Regression & 1.250 & 5 & .250 & 1.237 & $.307^{b}$ \\
\hline & Residual & 9.504 & 47 & .202 & & \\
\hline & Total & 10.755 & 52 & & & \\
\hline
\end{tabular}

a. Dependent Variable: Bank e-system Internet Payment

b. Predictors: (Constant), Internet Banking: Banks capacity, Internet Banking: improved Epayment security, Internet Banking: low costs of implementation, Internet Banking: low transaction risk, Internet Banking: Perceived usefulness
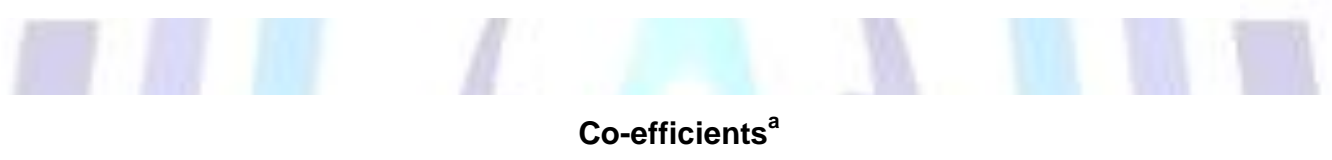

Co-efficients $^{\mathrm{a}}$

\begin{tabular}{|c|c|c|c|c|c|c|}
\hline \multirow{2}{*}{\multicolumn{2}{|c|}{ Model }} & \multicolumn{2}{|c|}{ Unstandardized Coefficients } & \multirow{2}{*}{$\begin{array}{c}\begin{array}{c}\text { Standardized } \\
\text { Coefficients }\end{array} \\
\text { Beta }\end{array}$} & \multirow[t]{2}{*}{$\mathrm{t}$} & \multirow[t]{2}{*}{ Sig. } \\
\hline & & B & Std. Error & & & \\
\hline \multirow{6}{*}{1} & (Constant) & .622 & .158 & & 3.927 & .000 \\
\hline & $\begin{array}{l}\text { Internet Banking: improved } \\
\text { E-payment security }\end{array}$ & -.015 & .138 & -.016 & -.106 & .916 \\
\hline & $\begin{array}{l}\text { Internet Banking: low costs } \\
\text { of implementation }\end{array}$ & -.078 & .140 & -.082 & -.559 & .579 \\
\hline & $\begin{array}{l}\text { Internet Banking: Perceived } \\
\text { usefulness }\end{array}$ & -.065 & .145 & -.069 & -.446 & .658 \\
\hline & $\begin{array}{l}\text { Internet Banking: low } \\
\text { transaction risk }\end{array}$ & -.165 & .133 & -.183 & -1.240 & .221 \\
\hline & $\begin{array}{l}\text { Internet Banking: Banks } \\
\text { capacity }\end{array}$ & .358 & .159 & .333 & 2.247 & .029 \\
\hline
\end{tabular}

a. Dependent Variable: Bank e-system Internet Payment

\section{CONCLUSION}

Banking industry in Kenya is undergoing technological evolution that is shaping business landscape extensively. From the study, banks are not sure on the most appropriate direction to take, that is, maintain status quo or shift their operations to the demands of digital generation lifestyle. Nevertheless, it is notable that ICT has made it possible to have electronic payment systems like debit cards, credit cards, electronic fund transfer, direct credits and internet banking a reality in banking sector. These new methods of transactions have made it easier for banks operations to cut on capital costs, reach many clients irrespective of distance, improve security while transacting large amounts of sums, easier financial packages mass customization with the end result being high revenue stream for the banks. 
Of course where benefits are involved, a few challenges will always pop up. The obstacles that banks should address in order to continue benefiting from e-payment systems relate to factors like lack of e-payment systems trust by majority of bankers, incidences of online fraud, difficulty in usability of some technologies hence need for highly trained technical staff, high transaction costs due to double taxations and fear for high risk investments. If these factors can be overcome by banks, cash transaction will automatically come down with high uptake of EPS.

\subsection{Recommendations}

E-payment systems are a congruent of many subsystems ranging from software, hardware and human resources. The success of such a system may not take a 'one-size-fits-all' approach. However, where appropriate steps are taken involving all stakeholders, a lot can be realized by banks from such a system. The stakeholders in mind include the banks, government, and telecommunication operators.

Commercial banks in Kenya should increase accuracy and update of their product description. This helps to decrease the doubts of consumers in e-transaction and proves the seriousness of e-electronic payment systems. More and improved guidelines/ functions for websites will help them convince and attract more online buyers. Banks diversification of payment, decreasing cash and increasing non-cash payment would stimulate electronic payment transactions. Equipping bank staff with right information and communication technology (ICT) skills would motivate the staff who will eventually support online transaction projects initiated by the management.

The government of Kenya should continue to promulgate necessary regulations to make e-commerce legal framework complete and encourage the development of electronic payment systems platforms. The legal framework would provide the desired transaction features like non-repudiation, security, anonymity, divisibility among others which will encourage banks to launch online-based products with hope of good response from the market. Certain official electronic payment benchmarks should be established to have official and sufficient statistics of this business at government level. Efforts, through proper government programming, must be put in to educate and create awareness to the public of online transactions and the benefits accruing from electronic payment systems. Regular and supportive policies emphasizing use of electronic payment should also be into place.

This is the era of information and communication technology. The leading concern of electronic revolution in this $21^{\text {st }}$ century is to establish and ensure a better, easy and comfortable way of management, communication and development with the use of information technology. Thus e-commerce has become a buzzword of present information technology (Laisuzzaman, Imran, Nahid, Amin, \& Alim, 2010). ICT has reshaped e-commerce beyond online shopping, online stock, bond transactions, buying and downloading software without ever going to a store. These activities involve electronic payment systems which are a backbone activity of banks and other financial institutions.

Since telecommunication network is a major player in implementation of e-payment systems, high speed, competitive international broadband access coupled with high density of local telecommunication facilities is essential for growth of e-commerce transactions backed by banks in Kenya.

\subsection{Further Research Suggestions}

Citing scholarly publications and recommendations regarding effectiveness and efficiency brought about by electronic payment systems, it will be prudent for upcoming researchers to concentrate on a particular e-payment system, for example, ATM and do further prodding that will provide insight on how to make it cost effective hence likely to attract mass adoption on the market.

Further research is also suggested to find out why many electronic payment systems fail at infancy stage. Take the case of DigiCash, and BitCoins, irrespective of their unique innovations, the technology could not sustain itself on the market.

\section{ACKNOWLEDGEMENT}

This research paper was presented to the University of Nairobi for the award of MBA Degree under the supervision of Dr. Kate Litondo, Lecturer School of Business, Department of Management Sciences - University of Nairobi. I really acknowledge her input. Thanks too to Mr. Kelvin Kabeti Omieno, Assistant Lecturer Department of Computer Science - Masinde Muliro University of Science and Technology for guiding me on where to publish my academic articles.

\section{REFERENCES}

[1] Aduda, J., \& Kingoo, N. (2012). The Relationship between Electronic Banking and Financial Performance among Commercial Banks in Kenya. Journal of Finance and Investment Analysis, 1(3), 99118.

[2] Akintoye, K. A., \& Araoye, O. I. (2011, July). Combating E-Fraud on Electronic Payment System. International Journal of Computer Applications, 25(8), 48-53.

[3] AL-Adwan, M. M., AL-Zyood, M., \& Ishfaq, M. (2013, January). The Impact of Electronic Payment on Saudi Banks. International Journal of Research and Reviews in Applied Sciences, 14(1), 100-113. 
Ayo, C. K., Adewoye, J. O., \& Oni, A. A. (2011, July 4). Business-to-consumer e-commerce in Nigeria: Prospects and Challenges. African Journal of Business Management, 5(13), 5109-5117.

[5] Bandyopadhyay, G. (2009). Banking the Unbanked: Going Mobile in Africa. Bangalore: Infosys Technologies.

[6] Birch, D. (2010, June 8). Making a silk e-purse. Retrieved April 17, 2013, from Digital Money: http://digitaldebateblogs.typepad.com/digital_money/2010/06/making-a-silk-e-purse.html

Bizina, E. (2012, February 19). Payment Systems for eCommerce Websites: Which One to Choose? Retrieved February 27, 2013, from Business2Community.com: http://www.business2community.com/tech-gadgets/payment-systems-for-ecommerce-websites-whichone-to-choose-0132530

[8] Bolt, W., Humphrey, D., \& Uittenbogaarda, R. (2008, March). Transaction Pricing and the Adoption of Electronic Payments: A Cross-Country Comparison. International Journal of Central Banking, 89-123.

[9] Boping, Z., \& Shiyu, S. (2009). An Improved SET Protocol. Proceedings of the 2009 International Symposium on Information Processing (pp. 267-272). Huangshan: Academy Publisher.

[10] Botha, J., Bothma, C., \& Geldenhuys, P. (2008). Managing E-commerce in Business (2 ed.). Cape Town: Juta.

[11] Boyd, C., \& Jacob, K. (2007). Mobile Financial Services for the Underbanked: Opportunities for Mbanking and M-payments. Chicago: Center for Financial Services Innovation.

[12] CBK. (2012). Bank Supervision Reports. Retrieved May 2, 2013, from Central Bank of Kenya: http://www.centralbank.go.ke/index.php/bank-supervision-reports

[13] Chaffey, D. (2009). E-Business and E-Commerce Management: Strategy, Implementation and Practice (4 ed.). Harlow, England: Pearson Education.

[14] Chaffey, D., \& Wood, S. (2005). Business Information Management: Improving Performance using Information Systems. Harlow, England: Prentice Hall.

[15] Chan, H., Lee, R., Dillon, T., \& Chang, E. (2001). E-Commerce: Fundamentals and Applications. Chichester: John Wiley \& Sons.

[16] Cooper, D. R., \& Schindler, P. S. (2006). Business Research Methods (9th ed.). New Delhi: Tata McGraw Hill Education.

[17] Cracknell, D. (2012). Policy Innovations to Improve Access to Financial Services in Developing Countries: Learning from Case Studies in Kenya. Nairobi: Center for Global Development.

[18] Dahlberg, T., Mallat, N., Ondrus, J., \& Zmijewska, A. (2007). Past, Present and Future of Mobile Payments Research: A Literature Review. Electronic Commerce Research and Applications, 1-17.

[19] Deans, P. C. (2005). E-commerce and M-commerce Technologies. Hershey: IRM.

[20] Deitel, P. J., \& Deitel, H. M. (2009). Java: How to Program (7 ed.). New Jersey: Prentice Hall.

[21] Delone, W. H., \& McLean, E. R. (2003). The DeLone and McLean Model of Information Systems Success: A Ten-Year Update. Journal of Management Information Systems, 9(4), 9-30.

[22] Dorsey, D., \& Jabob, K. (2005). Financial Services Trends and Recent Innovations in South Africa: Lessons for the United States. Chicago: Center for Financial Services Innovation.

[23] Đurić, Z., Marić, O., \& Gašević, D. (2007). Internet Payment System: A New Payment System for Internet Transactions. Journal of Universal Computer Science, 13(4), 479-503.

[24] European-Commission. (September 2005). ICT Security, e-Invoicing and e-Payment Activities in European Enterprises. Bonn: European Commission.

[25] Evans, D. (April 2011). San Jose: Cisco Internet Business Solutions Group.

[26] Gharegozi, A., Faraji, E., \& Heydari, L. (2011). The Study of Information Technology Effect on Ecommerce Growth. 2011 International Conference on Advancements in Information Technology (pp. 5054). Singapore: IACSIT Press.

[27] Gichane, C. (2012, October 10). Samsung, KWFT Pact to Boost Mobile Banking. Retrieved May 1, 2013, from Capital FM Business: http://www.capitalfm.co.ke/business/2012/10/samsung-kwft-pact-toboost-mobile-banking/

[28] Gikandi, J. W., \& Bloor, C. (2010). Adoption and Effectiveness of Electronic Banking in Kenya. Electronic Commerce Research and Applications, 9, 277-282. 
[29] Hai, M. (2010). A P2P E-Commerce Model Based on Interest Community. Management of e-Commerce and e-Government (ICMeCG), 2010 Fourth International Conference on (pp. 362-365). Beijing: Yan Tu.

[30] Harris, H., Guru, B. K., \& Avvari, M. V. (2011, December). Evidence of Firms' Perceptions Toward Electronic Payment Systems (EPS) in Malaysia. International Journal of Business and Information, 6(2), 226-245.

[31] Haseeb, K., Arshad, M., Ali, S., \& Yasin, S. (2011). Secure E-Commerce Protocol. International Journal of Computer Science and Security, 5(1), 132-142.

[32] Heffernan, S. (2005). Modern Banking. London: John Wiley \& Sons.

[33] Howitt, D., \& Cramer, D. (2011). Introduction to Research Methods in Psychology. Harlow, England: Prentice Hall.

[34] Indvik, L. (2011, February 28). Mashable. Retrieved February 7, 2013, from Forrester: E-Commerce To Reach Nearly \$300 Billion in U.S. by 2015: http://mashable.com/2011/02/28/forrester-e-commerce/

[35] Ingenico. (2012). Electronic Payment Architecture and Trends in Europe. Köln: Ingenico Corporate Communication.

[36] Jing, Y. (2009). On-line Payment and Security of E-commerce. Proceedings of the 2009 International Symposium on Web Information Systems and Applications (WISA'09) (pp. 46-50). Nanchang: Academy.

[37] Kawatra, N., \& Kumar, V. (2011). Analysis of E-Commerce Security Protocols SSL and SET. National Workshop-Cum-Conference on Recent Trends in Mathematics and Computing (pp. 1-5). Karnal: Guru Nanak Khalsa College.

[38] KCB. (2013, May 1). KCB to Boost M-Pesa Services. Retrieved May 1, 2013, from Kenya Commercial Bank: http://www.kcbbankgroup.com/ke/index.php?option=com_content\&task=view\&id=538\&ltemid=264

[39] Keller, K. L., Brady, M., Goodman, M., \& Hansen, T. (2009). Marketing Management. (P. Kotler, Ed.) Culemborg: Centraal Boekhuis.

[40] Khan, M. A. (2010). An Empirical Study of Automated Teller Machine Service Quality and Customer Satisfaction in Pakistani Banks. European Journal of Social Sciences, 13(3), 333-344.

[41] Kilonzo, K. D. (2007). An Analysis of the Legal Challenges posed by Electronic Banking. Kenya Law Review, 1, 323-341.

[42] Kim, H.-J., Mannino, M., \& Nieschwietz, R. J. (2009). Information Technology Acceptance in the Internal Audit Profession: Impact of Technology Features and Complexity. International Journal of Accounting Information Systems, 10, 214-228.

[43] Kimball, R. C. (1998, July/August). Economic Profit and Performance Measurement in Banking. New England Economic Review, 35-53.

[44] Kimwele, M., Mwangi, W., \& Kimani, S. (2010). Adoption of Information Technology Security Policies: Case Study of Kenyan Small and Medium Enterprises (SMES). Journal of Theoretical and Applied Information Technology, 1-11.

[45] Laisuzzaman, I. M., Imran, N., Nahid, A. A., Amin, M. Z., \& Alim, M. A. (2010). The Framework for Implementing E-commerce: The Role of Bank and Telecom in Bangladesh. Journal of Telecommunications, 1(1), 57-62.

[46] Laudon, K., \& Traver, C. (2007). E-Commerce Business, Technology, Society.

[47] Leonard, L. N. (2012). Attitude Influences in C2C E-commerce: Buying and Selling. Journal of Computer Information Systems, 11-17.

[48] Lule, I., Omwansa, T. K., \& Waema, T. M. (2012, June). Application of Technology Acceptance Model (TAM) in M-banking Adoption in Kenya. International Journal of Computing and ICT Research, 6(1), 31 . 43.

[49] Magutu, P. O., Mwangi, M., Nyaoga, R. B., Monchari, O. G., Kagu, M., Mutai, K., et al. (2011). E Commerce Products and Services in the Banking Industry: The Adoption and Usage in Commercial Banks in Kenya. Journal of Electronic Banking Systems, 1-19.

[50] Maravilla, N. (2013, 4 3). What Kinds of Products or Services Can You Sell Best on the Internet? Retrieved 4 3, 2013, from Power Home Business: http://www.powerhomebiz.com/vol12/kindsofproducts.htm

[51] Meenu, Pankaj, P. K., \& Nath, T. (2011). Enhanced Security in Secure Socket Layer 3.0 Specification. International Journal on Computer Science and Engineering, 3(9), 3259-3264. 
Mensah, A. O., Bahta, A., \& Mhlanga, S. (2005). E-commerce challenges in Africa: issues , constraints, opportunities. World Summit on the Information Society (pp. 1-4). Geneva: Economic Commission for Africa.

[53] Natarajan, T., Balasubramanian, S. A., \& Manickavasagam, S. (2010, August). Customer's Choice amongst Self Service Technology (SST) Channels in Retail Banking: A Study Using Analytical Hierarchy Process (AHP). Journal of Internet Banking and Commerce, 15(2), 1-16.

[54] Njuguna, P. K., Ritho, C., Olweny, T., \& Wanderi, M. P. (2012). Internet Banking Adoption in Kenya: The Case of Nairobi County. International Journal of Business and Social Science, 3(18), 246-252.

[55] Onyango, F. (2013, March 25). Bank Unlocks E-Commerce with Online Payment Portal. Retrieved March 25, 2013, from Business Daily: http://www.businessdailyafrica.com/Corporate-News//539550/846494/-/14x67h1z/-/index.html

[56] Parshotam, Cheema, R., \& Gulati, A. (2012, June). Improving the Secure Socket Layer by Modifying the RSA Algorithm. International Journal of Computer Science, Engineering and Applications, 3, 79-86.

[57] Pattnaik, S., Ghosh, P. P., \& Bharti, A. K. (2010). The Working Model of an E-paymeent System. Journal of Theoretical and Applied Information Technology, 10-13.

[58] Petter, S., DeLone, W., \& McLean, E. (2008). Measuring Information Systems Success:Models, Dimensions, Measures, and Interrelationships. European Journal of Information Systems, 17, 236-263.

[59] Pickens, M., Porteous, D., \& Rotman, S. (October 2005). Focus Note: Scenarios for Branchless Banking in 2020. London: Department for International Development.

[60] Pierzchala, S. (2013, March 16). The Revolution is Over, and M-Commerce is Here to Stay. Retrieved April 11, 2013, from E-Commerce Times: http://www.ecommercetimes.com/story/77553.html

[61] Porteous, D. (2006). The Enabling Environment for Mobile Banking in Africa. Boston: Department for International Development - DFID.

[62] Putland, P. A., Ward, C., Jackson, A., \& Trollope, C. (1999, July). Electronic Payment Systems. BT Technology, 17(3), 67-71.

[63] Rahul, R. (2012, April 6). Different types of E-commerce. Retrieved April 8, 2013, from Knowledge Tank: http://www.projectguru.in/publications/different-types-of-e-commerce/

[64] Rayport, J. F., \& Jaworski, B. J. (2004). Introduction to E-commerce. Boston: McGraw-Hill Irwin MarketspaceU.

[65] Rotman, S. (2011, July 20). The Cost-Benefit of Electronic Payment Systems: The Case of India. Retrieved April 3, 2013, from Advancing Financial Inclusion to Improve the Lives of the Poor: http://www.cgap.org/blog/cost-benefit-electronic-payment-systems-case-india

[66] Saunders, M., Lewis, P., \& Thornhill, A. (2009). Research Methods for Business Students (5th ed.). Harlow, England: Prentice Hall.

[67] Schneider, G. P. (2011). Electronic Commerce (9th ed.). Boston: Course Technology, CENGAGE Learning.

[68] Stallings, W. (2013, January 15). SSL: Foundation for Web Security. Retrieved April 23, 2013, from The Internet Protocol Journal - Volume 1, No. http://www.cisco.com/web/about/ac123/ac147/archived_issues/ipj_1-1/ssl.html

[69] Sumanjeet, S. (2009). Emergence of Payment Systems in the Age of Electronic Commerce: The State of Art. Global Journal of International Business Research, 2(2), 17-36.

[70] Taherdoost, H., Sahibuddin, S., \& Jalaliyoon, N. (2011). Smart Card Security; Technology and Adoption. International Journal of Security (IJS), 5(2), 74-84.

[71] Thanasankit, T. (2003). Ecommerce and Cultural Values. London: Idea Group.

[72] Thomas, I., Davie, W., \& Weidenhamer, D. (2013). Quarterly Retail E-commerce Sales 4th Quarter 2012. Washington DC: U.S. Census Bureau News.

[73] UNCTAD. (2005). Information Economy Report 2005. New York: United Nations.

[74] UNCTAD. (2011). ICTs as an Enabler for Private Sector Development. United Nations Conference on Trade and Development. New York: United Nations.

[75] Walliman, N. (2011). Research Methods: The Basics. London: Routledge.

[76] Wigder, Z. D. (2009, July 21). Forrester reports spike in Internet users. Retrieved February 7, 2013, from Boston Business Journal: http://www.bizjournals.com/boston/stories/2009/07/20/daily15.html 


\section{ISSN 2278-5612}

[77] Zhang, L., Yin, J., \& Li, M. (2006, August). Modeling Electronic Payment Systems Based on Dynamic Game. Journal of Communication and Computer, 3(8), 69-75.

[78] Zhu, D. (2002). Security Control in Inter-Bank Fund Transfer. Journal of Electronic Commerce Research, 3(1), 15-22.

[79] Zwass, V. (2003, Spring 2003). Electronic Commerce and Organizational Innovation: Aspects and Opportunities. International Journal of Electronic Commerce, 7(3), 7-37. 